\title{
Existence of nonoscillatory solutions for system of higher-order neutral differential equations with distributed coefficients and delays
}

Youjun Liu' ${ }^{1 *}$, Huanhuan Zhao' and Jurang Yan²

*Correspondence: lyj9791@126.com ${ }^{1}$ College of Mathematics and Computer Sciences, Shanxi Datong University, Datong, Shanxi 037009, P.R. China

Full list of author information is available at the end of the article

\begin{abstract}
In this paper we consider the existence of nonoscillatory solutions for a system of higher-order neutral differential equations with distributed coefficients and delays. We use the Banach contraction principle to obtain new sufficient conditions for the existence of nonoscillatory solutions.
\end{abstract}

Keywords: system; higher-order; distributed coefficients and delays; nonoscillatory solutions; Banach contraction principle

\section{Introduction and preliminary}

In this paper, we consider the system of higher-order neutral differential equations with distributed coefficients and delays

$$
\begin{aligned}
& {\left[r(t) \mathbf{x}(t)+\int_{a}^{b} p(t, \theta) \mathbf{x}(t-\theta) d \theta\right]^{(n)}} \\
& \quad+(-1)^{n+1}\left[\int_{c}^{d} Q_{1}(t, \tau) \mathbf{x}(t-\tau) d \tau-\int_{e}^{f} Q_{2}(t, \sigma) \mathbf{x}(t-\sigma) d \sigma-\mathbf{h}(t)\right]=\mathbf{0},
\end{aligned}
$$

(1) where $n$ is a positive integer, $n \geq 1,0<a<b, 0<c<d, 0<e<f$;

(2) $r \in C\left(\left[t_{0}, \infty\right), R^{+}\right), r(t)>0, p \in C\left(\left[t_{0}, \infty\right) \times[a, b], R\right), \mathbf{h} \in C\left(\left[t_{0}, \infty\right), \mathbf{R}\right)$,

(3) $\mathbf{x} \in \mathbf{R}^{n}, Q_{i}$ is continuous $n \times n$ matrix on $\left[t_{0}, \infty\right), i=1,2$.

Recently there have been a lot of activities concerning the existence of nonoscillatory solutions for neutral differential equations with positive and negative coefficients. In 2005, the existence of nonoscillatory solutions of the first-order linear neutral delay differential equations

$$
\frac{d}{d t}[x(t)+P(t) x(t-\tau)]+Q_{1}(t) x\left(t-\sigma_{1}\right)-Q_{2}(t) x\left(t-\sigma_{2}\right)=0
$$

(c) The Author(s) 2017. This article is distributed under the terms of the Creative Commons Attribution 4.0 International License (http://creativecommons.org/licenses/by/4.0/), which permits unrestricted use, distribution, and reproduction in any medium, provided you give appropriate credit to the original author(s) and the source, provide a link to the Creative Commons license, and indicate if changes were made. 
was investigated by Zhang et al. [1]. In 2012, Candan [2] studied the higher-order nonlinear differential equation

$$
\left[r(t)[x(t)+P(t) x(t-\tau)]^{(n-1)}\right]^{\prime}+(-1)^{n+1}\left[Q_{1}(t) g_{1}\left(x\left(t-\sigma_{1}\right)\right)-Q_{2}(t) g_{2}(x(t-\mu))-f(t)\right]=0 .
$$

In 2013, Candan [3] has investigated the existence of nonoscillatory solutions for the system of higher-order nonlinear neutral differential equations

$$
[\mathbf{x}(t)+P(t) \mathbf{x}(t-\theta)]^{(n)}+(-1)^{n+1}\left[Q_{1}(t) \mathbf{x}\left(t-\sigma_{1}\right)-Q_{2}(t) \mathbf{x}\left(t-\sigma_{2}\right)\right]=\mathbf{0} .
$$

In the same year, Liu et al. [4] has obtained the existence of nonoscillatory solutions for the system of higher-order neutral differential equations

$$
\begin{aligned}
& {\left[r(t)[\mathbf{x}(t)+P(t) \mathbf{x}(t-\theta)]^{(n-1)}\right]^{\prime}} \\
& \quad+(-1)^{n}\left(\int_{c}^{d} Q_{1}(t, \tau) \mathbf{x}(t-\tau) d \tau-\int_{e}^{f} Q_{2}(t, \sigma) \mathbf{x}(t-\sigma) d \sigma\right)=\mathbf{0} .
\end{aligned}
$$

As can be seen from the development process of the above equations, the delay of neutral part in the discussed differential equations were all constant delays. However, the case for distributed deviating arguments is rather rare; see [5, 6]. In 2015, Candan and Gecgel [6] studied the systems of higher-order neutral differential equations with distributed delay

$$
\begin{aligned}
& {\left[\left[x(t)+\int_{a_{3}}^{b_{3}} \tilde{P}(t, \xi) x(t-\xi) d \xi\right]\right]^{\prime}} \\
& \quad+(-1)^{n+1}\left[\int_{a_{1}}^{b_{1}} Q_{1}(t, \xi) \mathbf{x}(t-\xi) d \xi-\int_{a_{2}}^{b_{2}} Q_{2}(t, \xi) \mathbf{x}(t-\xi) d \xi\right]=\mathbf{0},
\end{aligned}
$$

the discussion only covered the condition for the coefficient being $0<\int_{a_{3}}^{b_{3}} \tilde{P}(t, \xi) x(t-$ $\xi) d \xi<\frac{1}{2}$ and $-\frac{1}{2}<\int_{a_{3}}^{b_{3}} \tilde{P}(t, \xi) x(t-\xi) d \xi<0$. However, in this paper, the difficulty in establishing a feasible operator was settled by skillful use of $r(t)$, and the coefficients $\int_{a}^{b} p_{2}(t, \xi) d \xi$ in the neutral part were all discussed in four cases, that is, $(-\infty,-1),(-1,0)$, $(0,1),(1,+\infty)$. Thus, in view of the above, this paper may have theoretical value as well as practical application value. For related work, we refer the reader to [7-10].

A solution of the system of equations (1) is a continuous vector function $\mathbf{x}(t)$ defined on $\left(\left[t_{1}-\mu, \infty\right), \mathbf{R}^{n}\right)$, for some $t_{1}>t_{0}$, such that $r(t) \mathbf{x}(t)-\int_{a}^{b} p(t, \theta) \mathbf{x}(t-\theta) d \theta$ is $n$ times continuously differentiable and the system of equations (1) holds for all $n \geq 1$. Here, $\mu=$ $\max \{b, \tau, \sigma\}$.

\section{The main results}

Theorem 1 Assume that $0 \leq \int_{a}^{b} p(t, \theta) d \theta \leq p_{1}<1$ and

$$
\begin{aligned}
& \int_{t_{0}}^{\infty} s^{n-1}\left\|\int_{c}^{d} Q_{1}(t, \tau) d \tau\right\| d s<\infty, \quad \int_{t_{0}}^{\infty} s^{n-1}\left\|\int_{e}^{f} Q_{2}(t, \sigma) d \sigma\right\| d s<\infty, \\
& \int_{t_{0}}^{\infty} s^{n-1}\|\mathbf{h}(s)\| d s<\infty
\end{aligned}
$$

Then equation (1) has a bounded nonoscillatory solution. 
Proof Let $\Lambda$ be the set of all continuous and bounded vector functions on $\left[t_{0}, \infty\right)$ with the sup norm. Set $A=\left\{x \in \Lambda, M_{1} \leq\|\mathbf{x}(t)\| \leq M_{2}, t \geq t_{0}\right\}$, where $M_{1}, M_{2}$ are two positive constants and $\mathbf{c}$ is a constant vector, such that $p_{1} M_{2}+\frac{M_{1}}{p_{1}}<\|\mathbf{c}\|<M_{2}, 1 \leq r(t) \leq \frac{1}{p_{1}}$. From (3), one can choose a $t_{1} \geq t_{0}, t_{1} \geq t_{0}+\mu$, sufficiently large, $t \geq t_{1}$, such that

$$
\begin{aligned}
& \int_{t}^{\infty} \frac{(s-t)^{n-1}}{(n-1) !}\left[M_{2}\left\|\int_{c}^{d} Q_{1}(t, \tau) d \tau\right\|+\|\mathbf{h}(s)\|\right] d s \leq M_{2}-\|\mathbf{c}\|, \\
& \int_{t}^{\infty} \frac{(s-t)^{n-1}}{(n-1) !}\left[M_{2}\left\|\int_{e}^{f} Q_{2}(s, \sigma) d \sigma\right\|+\|\mathbf{h}(s)\|\right] d s \leq\|\mathbf{c}\|-p_{1} M_{2}+\frac{M_{1}}{p_{1}}, \\
& \int_{t}^{\infty} \frac{(s-t)^{n-1}}{(n-1) !}\left[\left\|\int_{c}^{d} Q_{1}(t, \tau) d \tau\right\|+\left\|\int_{e}^{f} Q_{2}(s, \sigma) d \sigma\right\|\right] d s \leq 1-p_{1},
\end{aligned}
$$

and one defines an operator $T$ on $A$ as follows:

$$
(T \mathbf{x})(t)=\left\{\begin{array}{l}
\frac{1}{r(t)}\left\{\mathbf{c}-\int_{a}^{b} p(t, \theta) \mathbf{x}(t-\theta) d \theta+\int_{t}^{\infty} \frac{(s-t)^{n-1}}{(n-1) !}\left[\int_{c}^{d} Q_{1}(s, \tau) \mathbf{x}(s-\tau) d \tau\right.\right. \\
\left.\left.\quad-\int_{e}^{f} Q_{2}(s, \sigma) \mathbf{x}(s-\sigma) d \sigma-\mathbf{h}(s)\right] d s\right\} \quad t \geq t_{1}, \\
(T \mathbf{x})\left(t_{1}\right) \quad t_{0} \leq t \leq t_{1} .
\end{array}\right.
$$

It is easy to see that $T \mathbf{x}$ is continuous, for $t \geq t_{1}, \mathbf{x} \in A$, by using (4), we have

$$
\begin{aligned}
\|(T \mathbf{x})(t)\| & \leq \frac{1}{r(t)}\left\{\|\mathbf{c}\|+\left\|\int_{t}^{\infty} \frac{(s-t)^{n-1}}{(n-1) !}\left[\int_{c}^{d} Q_{1}(s, \tau) \mathbf{x}(s-\tau) d \tau-\mathbf{h}(s)\right] d s\right\|\right\} \\
& \leq\|\mathbf{c}\|+\int_{t}^{\infty} \frac{(s-t)^{n-1}}{(n-1) !}\left[M_{2}\left\|\int_{c}^{d} Q_{1}(t, \tau) d \tau\right\|+\|\mathbf{h}(s)\|\right] d s \\
& \leq M_{2}
\end{aligned}
$$

and taking (5) into account, we have

$$
\begin{aligned}
\|(T \mathbf{x})(t)\| \geq & \frac{1}{r(t)}\left\{\|\mathbf{c}\|-\int_{a}^{b} p(t, \theta)\|\mathbf{x}(t-\theta)\| d \theta\right. \\
& \left.-\left\|\int_{t}^{\infty} \frac{(s-t)^{n-1}}{(n-1) !}\left[\int_{e}^{f} Q_{2}(s, \sigma) \mathbf{x}(s-\sigma) d \sigma-\mathbf{h}(s)\right] d s\right\|\right\} \\
\geq & p_{1}\left\{\|\mathbf{c}\|-p_{1} M_{2}-\int_{t}^{\infty} \frac{(s-t)^{n-1}}{(n-1) !}\left(M_{1}\left\|\int_{e}^{f} Q_{2}(s, \sigma) d \sigma\right\|+\|\mathbf{h}(s)\|\right) d s\right\} \\
\geq & M_{1} .
\end{aligned}
$$

These show that $T A \subset A$, since $A$ is a bounded, closed, and convex subset of $\Lambda$, in order to apply the contraction principle we have to show that $T$ is a contraction mapping on $A$. For $\forall \mathbf{x}_{1}, \mathbf{x}_{2} \in A$, and $t \geq t_{1}$,

$$
\begin{aligned}
& \left\|\left(T \mathbf{x}_{1}\right)(t)-\left(T \mathbf{x}_{2}\right)(t)\right\| \\
& \leq \frac{1}{r(t)}\left\{\int_{a}^{b} p(t, \theta)\left\|\mathbf{x}_{1}(t-\theta)-\mathbf{x}_{2}(t-\theta)\right\| d \theta\right. \\
& \quad+\left\|\int_{t}^{\infty} \frac{(s-t)^{n-1}}{(n-1) !}\left[\int_{c}^{d} Q_{1}(s, \tau) \mathbf{x}_{1}(s-\tau) d \tau-\int_{e}^{f} Q_{2}(s, \sigma) \mathbf{x}_{1}(s-\sigma) d \sigma-\mathbf{h}(s)\right] d s\right\|
\end{aligned}
$$




$$
\begin{aligned}
& \left.-\left\|\int_{t}^{\infty} \frac{(s-t)^{n-1}}{(n-1) !}\left[\int_{c}^{d} Q_{1}(s, \tau) \mathbf{x}_{2}(s-\tau) d \tau-\int_{e}^{f} Q_{2}(s, \sigma) \mathbf{x}_{2}(s-\sigma) d \sigma-\mathbf{h}(s)\right] d s\right\|\right\} \\
\leq & \frac{1}{r(t)}\left\{p_{1}\left\|\mathbf{x}_{1}-\mathbf{x}_{2}\right\|+\int_{t}^{\infty} \frac{(s-t)^{n-1}}{(n-1) !}\left[\left\|\int_{c}^{d} Q_{1}(t, \tau) d \tau\right\|\left\|\mathbf{x}_{1}(s-\tau)-\mathbf{x}_{2}(s-\tau)\right\|\right.\right. \\
& \left.\left.+\left\|\int_{e}^{f} Q_{2}(s, \sigma) d \sigma\right\|\left\|\mathbf{x}_{1}(s-\sigma)-\mathbf{x}_{2}(s-\sigma)\right\|\right] d s\right\} .
\end{aligned}
$$

Using (6),

$$
\begin{aligned}
& \left\|\left(T \mathbf{x}_{1}\right)(t)-\left(T \mathbf{x}_{2}\right)(t)\right\| \\
& \quad \leq\left\|\mathbf{x}_{1}-\mathbf{x}_{2}\right\|\left(p_{1}+\int_{t}^{\infty} \frac{(s-t)^{n-1}}{(n-1) !}\left[\left\|\int_{c}^{d} Q_{1}(t, \tau) d \tau\right\|+\left\|\int_{e}^{f} Q_{2}(s, \sigma) d \sigma\right\|\right] d s\right) \\
& \quad<\left\|\mathbf{x}_{1}-\mathbf{x}_{2}\right\| .
\end{aligned}
$$

which shows that $T$ is a contraction mapping on $A$ and therefore there exists a unique solution, obviously a bounded positive solution of (1) $\mathbf{x} \in A$, such that $T \mathbf{x}=\mathbf{x}$. The proof is complete.

Theorem 2 Assume that $1<p_{3} \leq \int_{a}^{b} p(t, \theta) d \theta \leq p_{2}<2 p_{3}<+\infty$, and that (3) holds.

Then equation (1) has a bounded nonoscillatory solution.

Proof Let $\Lambda$ be the set of all continuous and bounded vector functions on $\left[t_{0}, \infty\right)$ with the sup norm. Set $A=\left\{x \in \Lambda, M_{3} \leq\|\mathbf{x}(t)\| \leq M_{4}, t \geq t_{0}\right\}$, where $M_{3}, M_{4}$ are two positive constants such that $p_{2} M_{4}+2 p_{2} M_{3}<\|\mathbf{c}\|<2 p_{3} M_{4}, 2 p_{3} \leq r(t) \leq 2 p_{2}$. From (3), one can choose a $t_{1} \geq t_{0}+b$, sufficiently large $t \geq t_{1}$, such that

$$
\begin{aligned}
& \int_{t}^{\infty} \frac{(s-t)^{n-1}}{(n-1) !}\left[M_{4}\left\|\int_{c}^{d} Q_{1}(s, \tau) d \tau\right\|+\|\mathbf{h}(s)\|\right] d s \leq 2 p_{3} M_{4}-\|\mathbf{c}\|, \\
& \int_{t}^{\infty} \frac{(s-t)^{n-1}}{(n-1) !}\left[M_{4}\left\|\int_{e}^{f} Q_{2}(s, \sigma) d \sigma\right\|+\|\mathbf{h}(s)\|\right] d s \leq\|\mathbf{c}\|-p_{2} M_{4}-2 p_{2} M_{3}, \\
& \int_{t}^{\infty} \frac{(s-t)^{n-1}}{(n-1) !}\left[\left\|\int_{c}^{d} Q_{1}(s, \tau) d \tau\right\|+\left\|\int_{e}^{f} Q_{2}(s, \sigma) d \sigma\right\|\right] d s \leq 2 p_{3}-p_{2},
\end{aligned}
$$

and one defines an operator $T$ on $A$ as follows:

$$
(T x)(t)=\left\{\begin{array}{l}
\frac{1}{r(t)}\left\{\mathbf{c}-\int_{a}^{b} p(t, \theta) \mathbf{x}(t-\theta) d \theta+\int_{t}^{\infty} \frac{(s-t)^{n-1}}{(n-1) !}\left[\int_{c}^{d} Q_{1}(s, \tau) \mathbf{x}(s-\tau) d \tau\right.\right. \\
\left.\left.\quad-\int_{e}^{f} Q_{2}(s, \sigma) \mathbf{x}(s-\sigma) d \sigma-\mathbf{h}(s)\right] d s\right\} \quad t \geq t_{1}, \\
(T \mathbf{x})\left(t_{1}\right) \quad t_{0} \leq t \leq t_{1} .
\end{array}\right.
$$

It is easy to see that $T$ is continuous, for $t \geq t_{1}, \mathbf{x} \in A$. By using (7), we have

$$
\begin{aligned}
\|(T \mathbf{x})(t)\| & \leq \frac{1}{r(t)}\left\{\|\mathbf{c}\|+\left\|\int_{t}^{\infty} \frac{(s-t)^{n-1}}{(n-1) !}\left[\int_{c}^{d} Q_{1}(s, \tau) \mathbf{x}(s-\tau) d \tau-\mathbf{h}(s)\right] d s\right\|\right\} \\
& \leq \frac{1}{2 p_{3}}\left\{\|\mathbf{c}\|+\int_{t}^{\infty} \frac{(s-t)^{n-1}}{(n-1) !}\left[M_{4}\left\|\int_{c}^{d} Q_{1}(s, \tau) d \tau\right\|+\|\mathbf{h}(s)\|\right] d s\right\} \leq M_{4},
\end{aligned}
$$


and taking (8) into account, we have

$$
\begin{aligned}
\|(T \mathbf{x})(t)\| \geq & \frac{1}{r(t)}\left\{\|\mathbf{c}\|-\int_{a}^{b} p(t, \theta)\|\mathbf{x}(t-\theta)\| d \theta\right. \\
& \left.+\left\|\int_{t}^{\infty} \frac{(s-t)^{n-1}}{(n-1) !}\left[\int_{e}^{f} Q_{2}(s, \sigma) \mathbf{x}(s-\sigma) d \sigma-\mathbf{h}(s)\right] d s\right\|\right\} \\
\geq & \frac{1}{2 p_{2}}\left\{\|\mathbf{c}\|-p_{2} M_{4}-\int_{t}^{\infty} \frac{(s-t)^{n-1}}{(n-1) !}\left(M_{4}\left\|\int_{e}^{f} Q_{2}(s, \sigma) d \sigma\right\|+\|\mathbf{h}(s)\|\right) d s\right\} \\
\geq & M_{3} .
\end{aligned}
$$

These show that $T A \subset A$, since $A$ is a bounded, closed, and convex subset of $\Lambda$, in order to apply the contraction principle, we have to show that $T$ is a contraction mapping on $A$. For $\forall \mathbf{x}_{1}, \mathbf{x}_{2} \in A$, and $t \geq t_{1}$,

$$
\begin{aligned}
&\left\|\left(T \mathbf{x}_{1}\right)(t)-\left(T \mathbf{x}_{2}\right)(t)\right\| \\
& \leq \frac{1}{r(t)}\left\{\int_{a}^{b} p(t, \theta)\left\|\mathbf{x}_{1}(t-\theta)-\mathbf{x}_{2}(t-\theta)\right\| d \theta\right. \\
& \quad+\left\|\int_{t}^{\infty} \frac{(s-t)^{n-1}}{(n-1) !}\left[\int_{c}^{d} Q_{1}(s, \tau) \mathbf{x}_{1}(s-\tau) d \tau-\int_{e}^{f} Q_{2}(s, \sigma) \mathbf{x}_{1}(s-\sigma) d \sigma-\mathbf{h}(s)\right] d s\right\| \\
&\left.\quad-\left\|\int_{t}^{\infty} \frac{(s-t)^{n-1}}{(n-1) !}\left[\int_{c}^{d} Q_{1}(s, \tau) \mathbf{x}_{2}(s-\tau) d \tau-\int_{e}^{f} Q_{2}(s, \sigma) \mathbf{x}_{2}(s-\sigma) d \sigma-\mathbf{h}(s)\right] d s\right\|\right\} \\
& \leq \frac{1}{r(t)}\left\{p_{2}\left\|\mathbf{x}_{1}-\mathbf{x}_{2}\right\|+\int_{t}^{\infty} \frac{(s-t)^{n-1}}{(n-1) !}\left[\left\|\int_{c}^{d} Q_{1}(s, \tau) d \tau\right\|\left\|\mathbf{x}_{1}(s-\tau)-\mathbf{x}_{2}(s-\tau)\right\|\right.\right. \\
&\left.\left.\quad+\left\|\int_{e}^{f} Q_{2}(s, \sigma) d \sigma\right\|\left\|\mathbf{x}_{1}(s-\sigma)-\mathbf{x}_{2}(s-\sigma)\right\|\right] d s\right\},
\end{aligned}
$$

using (9),

$$
\begin{aligned}
& \left\|\left(T \mathbf{x}_{1}\right)(t)-\left(T \mathbf{x}_{2}\right)(t)\right\| \\
& \quad \leq \frac{1}{2 p_{3}}\left\|\mathbf{x}_{1}-\mathbf{x}_{2}\right\|\left\{p_{2}+\int_{t}^{\infty} \frac{(s-t)^{n-1}}{(n-1) !}\left[\left\|\int_{c}^{d} Q_{1}(s, \tau) d \tau\right\|+\left\|\int_{e}^{f} Q_{2}(s, \sigma) d \sigma\right\|\right] d s\right\} \\
& \quad<\left\|\mathbf{x}_{1}-\mathbf{x}_{2}\right\|,
\end{aligned}
$$

which shows that $T$ is a contraction mapping on $A$ and therefore there exists a unique solution, obviously a bounded positive solution of (1) $\mathbf{x} \in A$, such that $T \mathbf{x}=\mathbf{x}$. The proof is complete.

Theorem 3 Assume that $-1<p_{4} \leq \int_{a}^{b} p(t, \theta) d \theta \leq 0$ and that (2) holds.

Then equation (1) has a bounded nonoscillatory solution.

Proof Let $\Lambda$ be the set of all continuous and bounded functions on $\left[t_{0}, \infty\right)$ with the sup norm. Set $A=\left\{x \in \Lambda, M_{5} \leq\|\mathbf{x}(t)\| \leq M_{6}, t \geq t_{0}\right\}$, where $M_{5}, M_{6}$ are two positive constants such that $\frac{M_{5}}{-p_{4}}<\|\mathbf{c}\|<\left(1+p_{4}\right) M_{6}, 1 \leq r(t) \leq \frac{1}{-p_{4}}$. From (3), one can choose a $t_{1} \geq t_{0}+b$, 
sufficiently large $t \geq t_{1}$ such that

$$
\begin{aligned}
& \int_{t}^{\infty} \frac{(s-t)^{n-1}}{(n-1) !}\left[M_{6}\left\|\int_{c}^{d} Q_{1}(s, \tau) d \tau\right\|+\|\mathbf{h}(s)\|\right] d s \leq\left(1+p_{4}\right) M_{6}-\|\mathbf{c}\|, \\
& \int_{t}^{\infty} \frac{(s-t)^{n-1}}{(n-1) !}\left[M_{6}\left\|\int_{e}^{f} Q_{2}(s, \sigma) d \sigma\right\|+\|\mathbf{h}(s)\|\right] d s \leq\|\mathbf{c}\|+\frac{M_{5}}{p_{4}}, \\
& \int_{t}^{\infty} \frac{(s-t)^{n-1}}{(n-1) !}\left[\left\|\int_{c}^{d} Q_{1}(s, \tau) d \tau\right\|+\left\|\int_{e}^{f} Q_{2}(s, \sigma) d \sigma\right\|\right] d s \leq 1+p_{4},
\end{aligned}
$$

and one defines an operator $T$ on $A$ as follows:

$$
(T \mathbf{x})(t)=\left\{\begin{array}{l}
\frac{1}{r(t)}\left\{\mathbf{c}-\int_{a}^{b} p(t, \theta) \mathbf{x}(t-\theta) d \theta+\int_{t}^{\infty} \frac{(s-t)^{n-1}}{(n-1) !}\left[\int_{c}^{d} Q_{1}(s, \tau) \mathbf{x}(s-\tau) d \tau\right.\right. \\
\left.\left.\quad-\int_{e}^{f} Q_{2}(s, \sigma) \mathbf{x}(s-\sigma) d \sigma-\mathbf{h}(s)\right] d s\right\} \quad t \geq t_{1}, \\
(T \mathbf{x})\left(t_{1}\right) \quad t_{0} \leq t \leq t_{1} .
\end{array}\right.
$$

It is easy to see that $T$ is continuous, for $t \geq t_{1}, \mathbf{x} \in A$. By using (10), we have

$$
\begin{aligned}
\|(T \mathbf{x})(t)\| \leq & \frac{1}{r(t)}\left\{\|\mathbf{c}\|-\int_{a}^{b} p(t, \theta)\|\mathbf{x}(t-\theta)\| d \theta\right. \\
& \left.+\left\|\int_{t}^{\infty} \frac{(s-t)^{n-1}}{(n-1) !}\left[\int_{c}^{d} Q_{1}(s, \tau) \mathbf{x}(s-\tau) d \tau-\mathbf{h}(s)\right] d s\right\|\right\} \\
\leq & \|\mathbf{c}\|-p_{4} M_{6}+\int_{t}^{\infty} \frac{(s-t)^{n-1}}{(n-1) !}\left[M_{6}\left\|\int_{c}^{d} Q_{1}(s, \tau) d \tau\right\|+\|\mathbf{h}(s)\|\right] d s \\
\leq & M_{6},
\end{aligned}
$$

and taking (11) into account, we have

$$
\begin{aligned}
& \|(T \mathbf{x})(t)\| \geq \frac{1}{r(t)}\left\{\|\mathbf{c}\|-\left\|\int_{t}^{\infty} \frac{(s-t)^{n-1}}{(n-1) !}\left[\int_{e}^{f} Q_{2}(s, \sigma) \mathbf{x}(s-\sigma) d \sigma-\mathbf{h}(s)\right] d s\right\|\right\} \\
& \quad \geq-p_{4}\left\{\|\mathbf{c}\|-\int_{t}^{\infty} \frac{(s-t)^{n-1}}{(n-1) !}\left[M_{6}\left\|\int_{e}^{f} Q_{2}(s, \sigma) d \sigma\right\|+\|\mathbf{h}(s)\|\right] d s\right\} \\
& \quad \geq M_{5} .
\end{aligned}
$$

These show that $T A \subset A$, since $A$ is a bounded, closed, and convex subset of $\Lambda$, in order to apply the contraction principle, we have to show that $T$ is a contraction mapping on $A$. For $\forall \mathbf{x}_{1}, \mathbf{x}_{2} \in A$, and $t \geq t_{1}$,

$$
\begin{aligned}
& \left\|\left(T \mathbf{x}_{1}\right)(t)-\left(T \mathbf{x}_{2}\right)(t)\right\| \\
& \leq \frac{1}{r(t)}\left\{\int_{a}^{b} p(t, \theta)\left\|\mathbf{x}_{1}(t-\theta)-\mathbf{x}_{2}(t-\theta)\right\| d \theta\right. \\
& \quad+\left\|\int_{t}^{\infty} \frac{(s-t)^{n-1}}{(n-1) !}\left[\int_{c}^{d} Q_{1}(s, \tau) \mathbf{x}_{1}(s-\tau) d \tau-\int_{e}^{f} Q_{2}(s, \sigma) \mathbf{x}_{1}(s-\sigma) d \sigma-\mathbf{h}(s)\right] d s\right\| \\
& \left.\quad-\left\|\int_{t}^{\infty} \frac{(s-t)^{n-1}}{(n-1) !}\left[\int_{c}^{d} Q_{1}(s, \tau) \mathbf{x}_{2}(s-\tau) d \tau-\int_{e}^{f} Q_{2}(s, \sigma) \mathbf{x}_{2}(s-\sigma) d \sigma-\mathbf{h}(s)\right] d s\right\|\right\}
\end{aligned}
$$




$$
\begin{aligned}
\leq & \frac{1}{r(t)}\left\{-p_{4}\left\|\mathbf{x}_{1}-\mathbf{x}_{2}\right\|+\int_{t}^{\infty} \frac{(s-t)^{n-1}}{(n-1) !}\left[\left\|\int_{c}^{d} Q_{1}(s, \tau) d \tau\right\|\left\|\mathbf{x}_{1}(s-\tau)-\mathbf{x}_{2}(s-\tau)\right\|\right.\right. \\
& \left.\left.+\left\|\int_{e}^{f} Q_{2}(s, \sigma) d \sigma\right\|\left\|\mathbf{x}_{1}(s-\sigma)-\mathbf{x}_{2}(s-\sigma)\right\|\right] d s\right\} .
\end{aligned}
$$

Or using (12),

$$
\begin{aligned}
& \left\|\left(T \mathbf{x}_{1}\right)(t)-\left(T \mathbf{x}_{2}\right)(t)\right\| \\
& \quad \leq\left\|\mathbf{x}_{1}-\mathbf{x}_{2}\right\|\left(-p_{4}+K L \int_{t}^{\infty} \frac{(s-t)^{n-1}}{(n-1) !}\left[\left\|\int_{c}^{d} Q_{1}(s, \tau) d \tau\right\|+\left\|\int_{e}^{f} Q_{2}(s, \sigma) d \sigma\right\|\right] d s\right) \\
& \quad<\left\|\mathbf{x}_{1}-\mathbf{x}_{2}\right\|,
\end{aligned}
$$

which shows that $T$ is a contraction mapping on $A$ and therefore there exists a unique solution, obviously a bounded positive solution of (1) $\mathbf{x} \in A$, such that $T \mathbf{x}=\mathbf{x}$. The proof is complete.

Theorem 4 Assume that $-\infty<2 p_{5}<p_{6} \leq \int_{a}^{b} p(t, \theta) d \theta \leq p_{5}<-1$ and that (3) holds.

Then equation (1) has a bounded nonoscillatory solution.

Proof Let $\Lambda$ be the set of all continuous and bounded functions on $\left[t_{0}, \infty\right)$ with the sup norm. Set $A=\left\{\mathbf{x} \in \Lambda, M_{7} \leq\|\mathbf{x}(t)\| \leq M_{8}, t \geq t_{0}\right\}$, where $M_{7}, M_{8}$ are two positive constants such that $-2 p_{6} M_{7}<\|\mathbf{c}\|<\left(-2 p_{5}+p_{6}\right) M_{8},-2 p_{5}<r(t)<-2 p_{6}$. From (3), one can choose a $t_{1} \geq t_{0}+b$, sufficiently large $t \geq t_{1}$, such that

$$
\begin{aligned}
& \int_{t}^{\infty} \frac{(s-t)^{n-1}}{(n-1) !}\left[M_{8}\left\|\int_{c}^{d} Q_{1}(s, \tau) d \tau\right\|+\|\mathbf{h}(s)\|\right] d s \leq-2 p_{5} M_{8}+p_{6} M_{8}-\|\mathbf{c}\|, \\
& \int_{t}^{\infty} \frac{(s-t)^{n-1}}{(n-1) !}\left[M_{8}\left\|\int_{e}^{f} Q_{2}(s, \sigma) d \sigma\right\|+\|\mathbf{h}(s)\|\right] d s \leq\|\mathbf{c}\|+2 p_{6} M_{7}, \\
& \int_{t}^{\infty} \frac{(s-t)^{n-1}}{(n-1) !}\left[\left\|\int_{c}^{d} Q_{1}(s, \tau) d \tau\right\|+\left\|\int_{e}^{f} Q_{2}(s, \sigma) d \sigma\right\|\right] d s \leq p_{6}-2 p_{5},
\end{aligned}
$$

and one defines an operator $T$ on $A$ as follows:

$$
(T \mathbf{x})(t)=\left\{\begin{array}{l}
\frac{1}{r(t)}\left\{\mathbf{c}-\int_{a}^{b} p(t, \theta) \mathbf{x}(t-\theta) d \theta+\int_{t}^{\infty} \frac{(s-t)^{n-1}}{(n-1) !}\left[\int_{c}^{d} Q_{1}(s, \tau) \mathbf{x}(s-\tau) d \tau\right.\right. \\
\left.\left.\left.\quad-\int_{e}^{f} Q_{2}(s, \sigma) \mathbf{x}(s-\sigma) d \sigma-\mathbf{h}(s)\right] d s\right]\right\} \quad t \geq t_{1} \\
(T \mathbf{x})\left(t_{1}\right) \quad t_{0} \leq t \leq t_{1} .
\end{array}\right.
$$

It is easy to see that $T$ is continuous, for $t \geq t_{1}, \mathbf{x} \in A$. By using (13), we have

$$
\begin{aligned}
\|(T \mathbf{x})(t)\| \leq & \frac{1}{r(t)}\left\{\|\mathbf{c}\|-\int_{a}^{b} p(t, \theta)\|\mathbf{x}(t-\theta)\| d \theta\right. \\
& \left.+\left\|\int_{t}^{\infty} \frac{(s-t)^{n-1}}{(n-1) !}\left[\int_{c}^{d} Q_{1}(s, \tau) \mathbf{x}(s-\tau) d \tau-\mathbf{h}(s)\right] d s\right\|\right\}
\end{aligned}
$$




$$
\begin{aligned}
& \leq \frac{1}{-2 p_{5}}\left\{\|\mathbf{c}\|-p_{6} M_{8}+\int_{t}^{\infty} \frac{(s-t)^{n-1}}{(n-1) !}\left[M_{8}\left\|\int_{c}^{d} Q_{1}(s, \tau) d \tau\right\|+\|\mathbf{h}(s)\|\right] d s\right\} \\
& \leq M_{8}
\end{aligned}
$$

and taking (14) into account, we have

$$
\begin{aligned}
\|(T \mathbf{x})(t)\| & \geq \frac{1}{r(t)}\left\{\|\mathbf{c}\|-\left\|\int_{t}^{\infty} \frac{(s-t)^{n-1}}{(n-1) !}\left[\int_{e}^{f} Q_{2}(s, \sigma) \mathbf{x}(s-\sigma) d \sigma-\mathbf{h}(s)\right] d s\right\|\right\} \\
& \geq \frac{1}{-2 p_{6}}\left\{\|\mathbf{c}\|-\int_{t}^{\infty} \frac{(s-t)^{n-1}}{(n-1) !}\left[M_{8}\left\|\int_{e}^{f} Q_{2}(s, \sigma) d \sigma\right\|+\|\mathbf{h}(s)\|\right] d s\right\} \\
& \geq M_{7} .
\end{aligned}
$$

These show that $T A \subset A$, since $A$ is a bounded, closed, and convex subset of $\Lambda$, in order to apply the contraction principle, we have to show that $T$ is a contraction mapping on $A$. For $\forall \mathbf{x}_{1}, \mathbf{x}_{2} \in A$, and $t \geq t_{1}$,

$$
\begin{aligned}
&\left\|\left(T \mathbf{x}_{1}\right)(t)-\left(T \mathbf{x}_{2}\right)(t)\right\| \\
& \leq \frac{1}{r(t)}\left\{\int_{a}^{b} p(t, \theta)\left\|\mathbf{x}_{1}(t-\theta)-\mathbf{x}_{2}(t-\theta)\right\| d \theta\right. \\
&+\left\|\int_{t}^{\infty} \frac{(s-t)^{n-1}}{(n-1) !}\left[\int_{c}^{d} Q_{1}(s, \tau) \mathbf{x}_{1}(s-\tau) d \tau-\int_{e}^{f} Q_{2}(s, \sigma) \mathbf{x}_{1}(s-\sigma) d \sigma-\mathbf{h}(s)\right]\right\| d s \\
&\left.-\left\|\int_{t}^{\infty} \frac{(s-t)^{n-1}}{(n-1) !}\left[\int_{c}^{d} Q_{1}(s, \tau) \mathbf{x}_{2}(s-\tau) d \tau-\int_{e}^{f} Q_{2}(s, \sigma) \mathbf{x}_{2}(s-\sigma) d \sigma-\mathbf{h}(s)\right] d s\right\|\right\} \\
& \leq \frac{1}{r(t)}\left\{-p_{6}\left\|\mathbf{x}_{1}-\mathbf{x}_{2}\right\|+\int_{t}^{\infty} \frac{(s-t)^{n-1}}{(n-1) !}\left[\left\|\int_{c}^{d} Q_{1}(s, \tau) d \tau\right\|\left\|\mathbf{x}_{1}(s-\tau)-\mathbf{x}_{2}(s-\tau)\right\|\right.\right. \\
&\left.\left.+\left\|\int_{e}^{f} Q_{2}(s, \sigma) d \sigma\right\|\left\|\mathbf{x}_{1}(s-\sigma)-\mathbf{x}_{2}(s-\sigma)\right\|\right] d s\right\} .
\end{aligned}
$$

Or using (15),

$$
\begin{aligned}
& \left\|\left(T \mathbf{x}_{1}\right)(t)-\left(T \mathbf{x}_{2}\right)(t)\right\| \\
& \leq \frac{1}{-2 p_{5}}\left\|\mathbf{x}_{1}-\mathbf{x}_{2}\right\|\left\{-p_{6}+\frac{1}{(n-1) !} \int_{t}^{\infty} \frac{(s-t)^{n-1}}{r(s)}\left[\left\|\int_{c}^{d} Q_{1}(s, \tau) d \tau\right\|\right.\right. \\
& \left.\left.\quad+\left\|\int_{e}^{f} Q_{2}(s, \sigma) d \sigma\right\|\right] d s\right\} \\
& <\left\|\mathbf{x}_{1}-\mathbf{x}_{2}\right\|,
\end{aligned}
$$

which shows that $T$ is a contraction mapping on $A$ and therefore there exists a unique solution, obviously a bounded positive solution of (1) $\mathbf{x} \in A$, such that $T \mathbf{x}=\mathbf{x}$. The proof is complete. 


\section{Example}

Consider the higher-order neutral differential equation with distributed coefficients and delays

$$
\begin{aligned}
& \left(\frac{2}{2+\sin t} \mathbf{x}(t)+\int_{\frac{\pi}{2}}^{\pi} e^{-t} \mathbf{x}(t-\theta) d \theta\right)^{(3)}+\int_{\frac{\pi}{2}}^{\frac{3 \pi}{2}} \tau e^{-t}\left(\begin{array}{cc}
\frac{5}{2} & \frac{7}{2} \\
5 & 1
\end{array}\right) \mathbf{x}(t-\tau) d \tau \\
& \quad-\int_{\pi}^{2 \pi} e^{-t}\left(\begin{array}{cc}
2 & 4 \\
\frac{7}{3} & \frac{11}{3}
\end{array}\right) \mathbf{x}(t-\sigma) d \sigma \\
& =e^{-t}\left(-4 \cos t-12 \pi \sin t-7 \pi+\pi^{2}\right)\left(\begin{array}{l}
1 \\
1
\end{array}\right) .
\end{aligned}
$$

Here, $n=3$,

$$
\begin{gathered}
r(t)=\frac{2}{2+\sin t}, \quad a=c=\frac{\pi}{2}, \quad b=e=\pi, \quad d=\frac{3 \pi}{2}, \\
f=2 \pi, \quad \mathbf{h}(t)=e^{-t}\left(-4 \cos t-12 \pi \sin t-7 \pi+\pi^{2}\right)\left(\begin{array}{l}
1 \\
1
\end{array}\right), \\
Q_{1}(t)=\int_{\frac{\pi}{2}}^{\frac{3 \pi}{2}} \tau e^{-t}\left(\begin{array}{cc}
\frac{5}{2} & \frac{7}{2} \\
5 & 1
\end{array}\right) d \tau, \quad Q_{2}(t)=\int_{\pi}^{2 \pi} e^{-t}\left(\begin{array}{cc}
2 & 4 \\
\frac{7}{3} & \frac{11}{3}
\end{array}\right) d \sigma .
\end{gathered}
$$

It is easy to see that $1 \leq r(t) \leq 2$,

$$
\begin{aligned}
& \int_{\frac{\pi}{2}}^{\pi} e^{-t} d \theta=\frac{\pi}{2} e^{-t}>1(t>0), \quad \int_{t}^{\infty} s^{2} \int_{\frac{\pi}{2}}^{\frac{3 \pi}{2}} \tau e^{-s}\left\|\left(\begin{array}{ll}
\frac{5}{2} & \frac{7}{2} \\
5 & 1
\end{array}\right)\right\| d \tau d s<\infty, \\
& \int_{t}^{\infty} s^{2} \int_{\pi}^{2 \pi} e^{-s}\left\|\left(\begin{array}{cc}
2 & 4 \\
\frac{7}{3} & \frac{11}{3}
\end{array}\right)\right\| d \sigma d s<\infty, \\
& \int_{t}^{\infty} s^{2} e^{-s}\left(-4 \cos s-12 \pi \sin s-7 \pi+\pi^{2}\right)\left\|\left(\begin{array}{l}
1 \\
1
\end{array}\right)\right\| d s<\infty,
\end{aligned}
$$

thus Theorem 2 holds.

In fact, $\mathbf{x}(t)=\left(\begin{array}{c}2+\sin t \\ 2+\sin t\end{array}\right)$ is a nonoscillatory solution of equation (16).

\section{Remark}

When $r(t) \equiv 1, \mathbf{h}(t)=\mathbf{0}$, equation (1) becomes equation (2), thus this paper improves results of Candan and Gecgel [6].

\section{Competing interests}

The authors declare that there is no conflict of interests regarding the publication of this article.

\section{Authors' contributions}

All authors contributed equally to the writing of this paper. All authors read and approved the final manuscript.

\section{Author details}

'College of Mathematics and Computer Sciences, Shanxi Datong University, Datong, Shanxi 037009, P.R. China. ${ }^{2}$ School of Mathematical Sciences, Shanxi University, Taiyuan, Shanxi 030006, P.R. China. 


\section{Acknowledgements}

This research is supported by Scientific Research Project Shanxi Datong University (No. 2011K3).

Received: 12 July 2016 Accepted: 7 December 2016 Published online: 07 February 2017

\section{References}

1. Zhang, W, Feng, W, Yan, J, Song, J: Existence of nonoscillatory solutions of first-order linear neutral delay differential equations. Comput. Math. Appl. 49, 1021-1027 (2005)

2. Candan, T: The existence of nonoscillatory solutions of higher order nonlinear neutral differential equations. Appl. Math. Lett. 25, 412-416 (2012)

3. Candan, T: Existence of nonoscillatory solutions for system of higher order neutral differential equations. Math. Comput. Model. 57, 375-381 (2013)

4. Liu, Y, Zhang, J, Yan, J: Existence of nonoscillatory solutions for system of higher-order neutral differential equations with distributed deviating arguments. Discrete Dyn. Nat. Soc. 2013, 1-8 (2013)

5. Candan, T: Existence of nonoscillatory solutions of first-order nonlinear neutral differential equations. Appl. Math. Lett. 26, 1182-1186 (2013)

6. Candan, T, Gecgel, AM: Existence of nonoscillatory solutions for system of higher order neutral differential equations with distributed delay. J. Comput. Anal. Appl. 18, 266-276 (2015)

7. Candan, T, Dahiya, RS: Existence of nonoscillatory solutions of first and second order neutral differential equations with distributed deviating arguments. J. Franklin Inst. 347, 1309-1316 (2010)

8. Liu, Y, Zhang, J, Yan, J: Existence of nonoscillatory solutions of higher order neutral differential equations with distributed deviating arguments. Acta Math. Appl. Sin. 38(2), 235-243 (Chinese) (2015)

9. Zhou, Y, Zhang, BG: Existence of nonoscillatory solutions of higher-order neutral differential equations with positive and negative coefficients. Appl. Math. Lett. 15, 867-874 (2002)

10. Györi, I, Ladas, G: Oscillation Theory of Delay Differential Equations with Applications. Clarendon Presss, Oxford (1991)

\section{Submit your manuscript to a SpringerOpen ${ }^{\circ}$ journal and benefit from:}

- Convenient online submission

- Rigorous peer review

- Immediate publication on acceptance

- Open access: articles freely available online

- High visibility within the field

- Retaining the copyright to your article 